\title{
Loss of the BH3-only protein Bid does not rescue RelA-deficient embryos from TNF-R1-mediated fatal hepatocyte destruction
}

\author{
Cell Death and Differentiation (2007) 14, 637-639. doi:10.1038/sj.cdd.4402061; published online 10 November 2006
}

\section{Dear Editor}

The BH3-only members of the Bcl-2 protein family are essential initiators of developmentally programmed cell death and stress-induced apoptosis. ${ }^{1}$ They act as molecular sensors for a variety of intracellular stress signals and are activated by transcriptional and/or post-translational mechanisms. BH3-only proteins require Bax/Bak-like proteins to transmit the apoptotic signal, but it remains unclear whether they activate Bax/Bak directly or do so indirectly by binding to prosurvival $\mathrm{Bcl}-2$ family members, thereby unleashing Bax/Bak. ${ }^{1}$

Bid is an unusual $\mathrm{BH} 3-$ only protein because it can be activated through proteolytic cleavage by caspases or certain other proteases (e.g. granzyme B). For example, during Fas (APO-1/CD95)-induced apoptosis signalling, full-length (relatively poorly active) Bid (p22) is processed by caspase- 8 into the potently proapoptotic, truncated tBid (p15), which translocates to the outer mitochondrial membrane where it promotes the release of apoptogenic molecules, such as cytochrome c, and amplification of the caspase cascade. ${ }^{2,3}$ Experiments with gene-targeted mice have shown that Bid is essential for anti-Fas antibody-induced apoptosis of hepatocytes in vivo. ${ }^{4}$ These mice had a mixed genetic background and retained a strong promoter (from the drug selection cassette) within the targeted locus, which can cause nonspecific effects owing to influences on expression of neighbouring genes. To obviate such potential problems, we have generated a new bid $^{-1-}$ mouse strain on a pure C57BL/6 genetic background and have removed the drug selection cassette and its strong promoter by Cre-mediated recombination (TK and AS, submitted). We confirmed that Bid is essential for anti-Fas antibody-induced fatal hepatitis and could also show that Bid is required for hepatocyte apoptosis induced by the more physiological recombinant FasL (TK and AS, submitted). This is an important finding because anti-Fas antibodies do not always reliably mimic the action of the physiological ligand. ${ }^{5}$ The role of Bid in TNF-TNF-R-mediated cell killing is, however, presently still unresolved.

In addition to the proapoptotic signalling pathway, ligation of TNF-R1 on hepatocytes and certain other cell types (e.g. fibroblasts) also triggers potent prosurvival as well as differentiation- and proliferation-inducing pathways. ${ }^{6,7}$ This involves, at least in part, activation of NF- $\kappa$ B transcription factors, such as p65/RelA and, accordingly, TNF-mediated apoptosis of fibroblasts is strongly enhanced by blocking this transcription-dependent, prosurvival pathway. ${ }^{8,9}$ In a wellestablished genetic mouse model, loss of p65/RelA leads to embryonic lethality around E15 owing to massive hepatocyte apoptosis. ${ }^{10}$ This death can be prevented by concomitant loss of TNF or TNF-R1, demonstrating that it is triggered by the TNF-TNF-R1 cell killing pathway. ${ }^{11,12}$ We have recently found that overexpression of $\mathrm{Bcl}-2$ in hepatocytes under control of the liver-specific $\alpha$-anti-trypsin (AAT) promoter is unable to prevent or even delay the fatal hepatocyte death in embryos lacking RelA. ${ }^{13}$ This may indicate that proapoptotic BH3-only proteins, such as Bid, are not critical for TNF-TNF-R1induced apoptosis in hepatocytes. However, binding studies have indicated that $\mathrm{Bcl}-2$ might not be the optimal neutralising interaction partner for truncated Bid (tBid), with the Bcl-2 homologues $\mathrm{Bcl}-\mathrm{x}_{\mathrm{L}}$ and $\mathrm{Bcl}-\mathrm{w}$ having much higher binding affinities for $\mathrm{tBid}^{14}{ }^{14}$ Consistent with the notion that $\mathrm{Bcl}-2$ is a poor antagonist of Bid, bid ${ }^{-1}$ mice, ${ }^{4}$ but not the AAT-bcl-2 transgenic mice ( ${ }^{5}$ and $\mathrm{TK}$ and AS, not shown), expressing $\mathrm{Bcl}-2$ in hepatocytes, are protected from anti-Fas antibody (Jo2) or FasL-induced fatal hepatitis.

In order to clarify this issue and to examine the importance of Bid in TNF-TNF-R1 cell death signalling, we intercrossed bid $^{-1-}$ with rela ${ }^{-1-}$ mice. Strikingly, the absence of Bid failed to rescue RelA-deficient embryos from death, as no viable bid $^{-1-}$ rela $^{-/-}$mice were observed among 182 offspring resulting from $\mathrm{bid}^{-1-} \mathrm{rela}^{+/-}$intercrosses (Figure 1a). Moreover, at $\mathrm{E} 14.5$, as well as at E15.5 $\mathrm{bid}^{-1-} \mathrm{rela}^{-1-}$ embryos showed a comparable extent of liver destruction and haemorrhaging as seen in control bid $^{+/+}$rela $^{-1-}$ embryos (Figure $1 \mathrm{~b}$ and c; see also Gugasyan et $a^{13}$ ). These results demonstrate that, in contrast to Fas-induced apoptosis, Bid is not essential for TNF-TNF-R1 signalling-mediated hepatocyte killing during embryonic development, at least within the context of RelAdeficiency.

Collectively, our results demonstrate that the proapoptotic FasL-Fas pathway differs from the TNF-TNF-R1 cell killing pathway, at least in mouse hepatocytes. Whereas Fasmediated apoptosis requires the $\mathrm{BH} 3$-only protein Bid, loss of Bid failed to prevent TNF-TNF-R1-mediated hepatocyte killing in RelA-deficient embryos. Hence, TNF-R1 must trigger cell death through components other than or in addition to Bid. Although differences between foetal and adult hepatocytes cannot be excluded, it is noteworthy that we found that adult $\mathrm{bid}^{-1-}$ mice are normally sensitive to TNF-mediated hepatocyte destruction (TK and AS, submitted). Moreover, although it is theoretically possible that non-apoptotic, caspase-independent processes mediate TNF-induced hepatocyte killing, as described in certain other cell types, ${ }^{7,15}$ loss of caspase-8 in hepatocytes protected mice against TNFmediated hepatitis (TK and AS, submitted). We therefore assume that TNF-TNF-R1 signalling kills RelA-deficient hepatocytes by caspase-dependent apoptosis. It is possible 
a

\begin{tabular}{ccc:c} 
Genotype & E14.5 & E15.5 & weaning (expected) \\
\hline bid $^{-/-}$rela $^{+/+}$ & 5 & 4 & $75(45.5)$ \\
\hline bid $^{-/-}$rela $^{+/-}$ & 11 & 7 & $107(91)$ \\
\hline bid $^{-/-}$rela $^{-/-}$ & 5 & 4 & $0(45.5)$ \\
\hline total & 21 & 15 & 182
\end{tabular}

b

E14.5

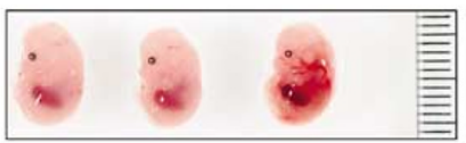

bid: $\quad-/-$

rela: +/-

c

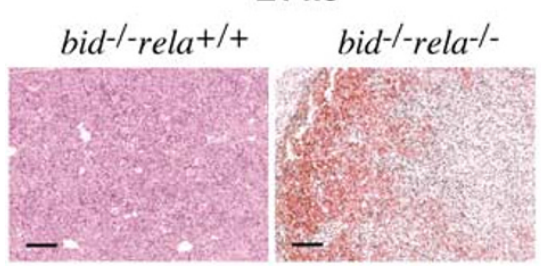

\section{E15.5}

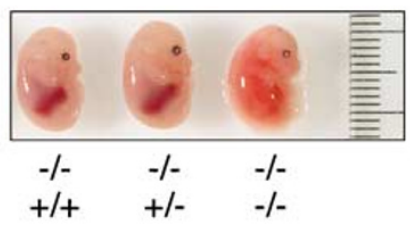

E15.5

Figure 1 Loss of Bid does not rescue RelA-deficient embryos from fatal hepatocyte destruction. (a) Genotyping results from offspring (at 3 weeks of age) and embryos (E14.5 and E15.5) from intercrosses of bid ${ }^{-l-} \mathrm{rela}^{+l-}$ mice. Whereas at all embryonic stages examined, a Mendelian distribution of genotypes was observed, none out of a total of 182 viable mice was found to be deficient for both Bid and RelA ( bid $^{-1-}$ rela ${ }^{-l-}$ ). This indicates that the absence of Bid does not rescue the lethality of RelAdeficient embryos. Numbers in brackets indicate the number of offspring for an expected Mendelian distribution. (b) Full body images of embryos of the indicated genotypes at E14.5 and E15.5. Note the extensive haemorrhaging in the livers of bid ${ }^{-1}$ rela $^{-1-}$ embryos at E14.5 and E15.5. (c) Histological examination of H\&Estained liver sections from E14.5 and E15.5 embryos of the indicated genotypes (bars $=50 \mu \mathrm{m}$ ). Pictures shown are representative of the analysis of at least four embryos for each genotype and stage of development. The generation of Bid-deficient mice is described elsewhere (TK and AS, submitted). The rela ${ }^{-1-}$ mice were originally generated on a mixed C57BL/6x129SV background using $129 \mathrm{SV}$-derived ES cells ${ }^{10}$ but had been backcrossed with C57BL/6 mice for $>9$ generations before intercrossing with our bid ${ }^{-1-}$ mice. All experiments with mice were carried out according to the guidelines of the animal ethics committees of the Melbourne Health Research Directorate

that TNF-R1 ligation stimulates levels of caspase-8 activity sufficient to trigger hepatocyte destruction, whereas Fas signalling elicits only relatively low levels of caspase-8 activity, therefore needing Bid-mediated amplification of the caspase cascade to kill cells. This hypothesis can be tested by generating RelA-deficient mice lacking caspase- 8 selectively in hepatocytes. It is also possible that TNF-R1 stimulation causes activation of $\mathrm{BH} 3-o n l y$ proteins or other proapoptotic factors in addition to Bid. Bim is a possible candidate, because it was reported to be activated in response to phosphorylation by $\mathrm{JNK}^{16}$ a kinase that is stimulated during TNF-R1 signalling. ${ }^{6}$ Moreover, caspase-mediated proteolysis has been shown to enhance the proapoptotic activity of $\operatorname{Bim}_{\mathrm{EL}},{ }^{17}$ the most highly expressed isoform of Bim in the liver, ${ }^{18,19}$ thereby creating an amplification of the apoptotic signalling cascade similar to the one involving Bid. It will therefore be interesting to test whether combined loss of Bid and Bim can prevent the fatal hepatocyte destruction in RelA-deficient embryos.

Acknowledgements. We are grateful to Drs. A Beg and D Baltimore for gifts of RelA knockout mice. We thank $N$ lannarella and $G$ Siciliano for animal care and $M$ Robati and $B$ Helbert for help with genotyping. This work was supported by grants (program \#257502) and fellowships from the NHMRC (Canberra), the $\mathrm{NCl}$ (NIH, USA; \# CA 80188 and \#CA 43540), the Leukemia and Lymphoma Society of America (SCOR grant; \#7015), the JDRF/NHMRC and the Swiss National Science Foundation, the Roche Research Foundation and the Novartis Foundation (formerly Ciba-Geigy-Jubilaeumsstiftung; Postdoctoral Fellowships to TK).

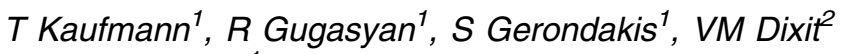 and $A$ Strasser ${ }^{*, 1}$}

1 The Walter and Eliza Hall Institute of Medical Research, Parkville, Melbourne, Victoria, Australia;

2 Genentech Inc., South San Francisco, CA, USA

* Corresponding author: A Strasser, Molecular Genetics of Cancer Division, The Walter and Eliza Hall Institute of Medical Research, 1G Royal Parade, Parkville, Melbourne, Victoria 3050, Australia. Tel: + 6139345 2555; Fax: + 61393470852 . E-mail: strasser@wehi.edu.au

\footnotetext{
1. Huang DCS, Strasser A. Cell 2000; 103: 839-842.

2. Luo $X$ et al. Cell 1998; 94: 481-490.

3. Li $\mathrm{H}$ et al. Cell 1998; 94: 491-501.

4. Yin X-M et al. Nature 1999; 400: 886-891.

5. Huang DC et al. Proc Natl Acad Sci USA 1999; 96: 14871-14876.

6. Aggarwal BB. Nat Rev Immunol 2003; 3: 745-756.

7. Meylan E, Tschopp J. Trends Biochem Sci 2005; 30: 151-159.

8. Wang C-Y et al. Science 1996; 274: 784-787.
} 
9. Beg AA, Baltimore D. Science 1996; 274: 782-784.

10. Beg AA et al. Nature 1995; 376: 167-170.

11. Doi TS et al. Proc Natl Acad Sci USA 1999; 96: 2994-2999.

12. Rosenfeld ME, Prichard L, Shiojiri N, Fausto N. Am J Pathol 2000; 156: 997-1007.

13. Gugasyan R et al. Cell Death Differ 2006; 13: 1235-1237.

14. Chen L et al. Mol Cell 2005; 17: 393-403.
15. Vanden Berghe T et al. J Biol Chem 2004; 279: 7925-7933.
16. Lei K, Davis RJ. Proc Natl Acad Sci USA 2003; 100: 2432-2437.
17. Chen D, Zhou Q. Proc Natl Acad Sci USA 2004; 101: 1235-1240.
18. O'Connor L et al. EMBO J 1998; 17: 384-395.
19. O'Reilly LA et al. Am J Pathol 2000; 157: 449-461. 